\title{
Genotyping of IRGM tetranucleotide promoter oligorepeats by fluorescence resonance energy transfer
}

\author{
Christopher D. Intemann ${ }^{1,2}$, Thorsten Thye ${ }^{1,2}$, Jürgen Sievertsen ${ }^{1}$, \\ Ellis Owusu-Dabo ${ }^{3,4}$, Rolf D. Horstmann ${ }^{1}$, and Christian G. Meyer ${ }^{1}$ \\ ${ }^{1}$ Bernhard Nocht Institute for Tropical Medicine, Department of Molecular Medicine, \\ Hamburg, Germany, 2 University Hospital Schleswig-Holstein, Campus Lübeck, \\ Institute of Medical Biometry and Statistics, Lübeck, Germany, ${ }^{3}$ Department of \\ Community Health, College of Health Sciences, Kwame Nkrumah University of \\ Science and Technology, Kumasi, Ghana, and ${ }^{4}$ Kumasi Centre for Collaborative \\ Research in Tropical Medicine, Kumasi, Ghana
}

BioTechniques 46:58-60 (January 2009)

doi $10.2144 / 000113045$

Fluorescence resonance energy transfer (FRET) genotyping has been well established for the rapid assessment of single nucleotide polymorphisms (SNPs) and deletions. A design is presented that allows the typing of short tandem oligo repeat sequences using the LightTyper/ LightCycler system. The protocol was evaluated and applied to the typing of a tetranucleotide promoter repeat of the human gene encoding the immunity-related GTPase family, $M$ (IRGM) molecule in >2000 individuals from Ghana, West Africa.

In addition to large-scale genetic association studies aimed at typing hundreds of thousands of single nucleotide polymorphisms (SNPs), smaller-scale projects focus on typing selected candidate SNPs. As large numbers of DNA samples have to be typed to assign genetic differences to distinct phenotypes, efficient high-throughput methods have to be employed. The Roche LightTyper and the LightCycler 480 Real-Time PCR instruments (Roche Diagnostics $\mathrm{GmbH}$, Mannheim, Germany) have been developed for detection of SNPs, and protocols have been provided for the typing of deletions. LightTyper/ LightCycler-based analyses can be performed rapidly with either 96- or 384-well microtiter plates (1), and thousands of SNP calls can be achieved per day at a reasonable cost (2).

Both systems allow post-amplification genotyping by fluorescence resonance energy transfer (FRET) and high-resolution DNA melting analysis with perfectly matched and discretely mismatched probes. The danger of contamination is minimized, as all reagents-including fluorescently labeled probes-are added to the microtiter plate at the reaction start. In addition, the FRET technology has been established and evaluated for the determination of DNA tetraplexes $(3,4)$. The LightTyper and the LightCycler devices consist of a temperature-regulated heat block and a high-resolution charge-coupled device (CCD) camera. While the reaction mixture undergoes slow and continuous heating at steadily increasing temperature ramps, diodes emit blue light to excite the labeled probes in the LightTyper system (475-nm excitation filter), and fluorescence emission is recorded by the CCD camera (600-nm emission interference longpass filter). In the LightCycler system, excitation is achieved by a Xenon reflector lamp (483-nm excitation filter; 670-nm emission filter). Samples are grouped according to the melting temperature $\left(\mathrm{T}_{\mathrm{m}}\right)$ and curve shapes, and scored in relation to an internal standard. Melting analyses are performed by the Roche LightTyper Software, Version 1.5.

Tandem repeat sequences are patchily disseminated throughout proand eukaryotic genomes and frequently exhibit a high degree of polymorphism. Among the techniques that have successfully been applied for the typing of such repeats are gel electrophoresis and capillary arrays (5-7), microchip capillary electrophoresis (8), mass spectrometry $(9,10)$, and hybridization arrays (11). High-throughput melting analyses of PCR-amplified repeats would, for several reasons, be an interesting alternative and the need to develop appropriate protocols has recently been addressed (12). While significantly expediting analyses, it would reduce the danger of contamination, but also the expense of assays.

We have developed a LightTyper/ LightCycler-based assay for rapid assessment of a tetranucleotide GTTT-repeat that occurs in the promoter of the human gene encoding the immunity-related GTPase family, M (IRGM) molecule (IRGM; OMIM *608212, gene map locus 5q33.1; rs60800371, position -308 to -311 relative to the ATG start codon), which plays a role in autophagy. We genotyped 2263 individuals originating from Ghana, West Africa, in a case-control study on susceptibility to pulmonary tuberculosis (TB). DNA was isolated from blood samples of study participants (AGOWA mag Maxi DNA Isolation Kit; AGOWA, Berlin, Germany) according to the manufacturer's instructions. In order to detect all IRGM variants occurring with a statistically high probability in our African study population, bidirectional resequencing of $1000 \mathrm{bp}$ upstream of the ATG start codon (ABI Prism 6100; Applied Biosystems, Foster City, USA), 775 coding bp, and 1053 bp of the first intron of the IRGM gene was performed in 69 individuals (23 TB cases, 23 healthy controls with a positive tuberculin response, and 23 healthy controls with a negative tuberculin response). Sequence alignment showed that the GTTT repeat occurred as $\mathrm{GTTT}_{1}, \mathrm{GTTT}_{3}$, and $\mathrm{GTTT}_{4}$ alleles. The GTTT $_{3}$ variant had not yet been recognized.

Amplification primers and sensor/ anchor (S/A) oligonucleotides were designed with Roche LightCycler ProbeDesign, Version 2.0.R.33. The sensor oligonucleotide perfectly matched the sequence of the $\mathrm{GTTT}_{4}$ 16-bp repeat with an overlap of $15 \mathrm{bp}$ in the $5^{\prime}$ orientation and $9 \mathrm{bp}$ in the $3^{\prime}$ 


\section{Benchmarks}

A

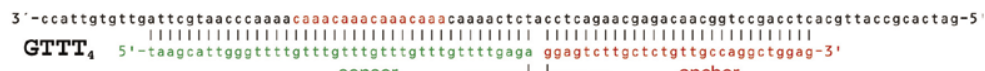

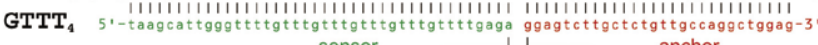

anchor

GTTT
Sensor

B $\quad \operatorname{GTTT}_{1 / 1}$

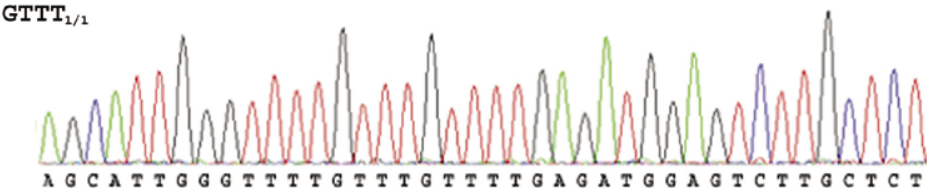

$\operatorname{GTTT}_{1 / 3}$

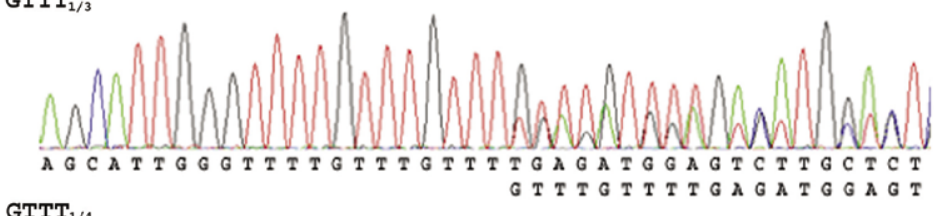
$\mathrm{GTTT}_{1 / 4}$

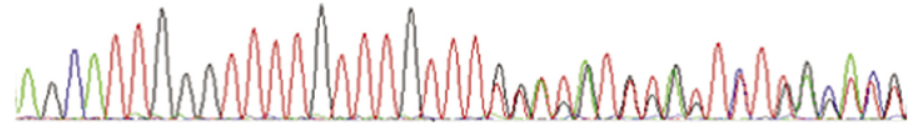

A G C A T TGGGTTTTGTTTGTTTTGAGATGGAGTCTTGCTCT G T T T G T T T G T T T T G A A T G $\operatorname{GTTT}_{3 / 3}$

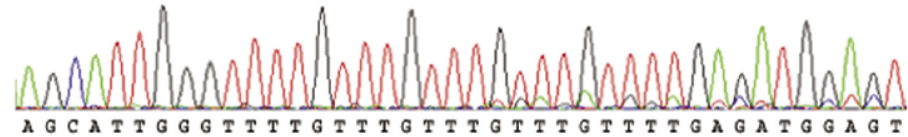

$\mathrm{GTTT}_{3 / 4}$

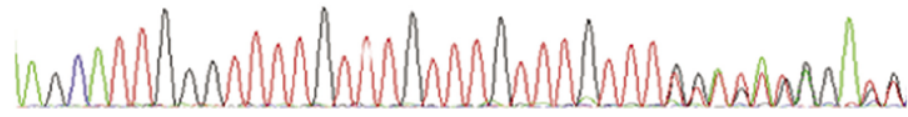

AGCATTGGGTTTTETTGTTTGTTTGTTTTGAGATGGAGT $\mathrm{GTTT}_{4 / 4}$

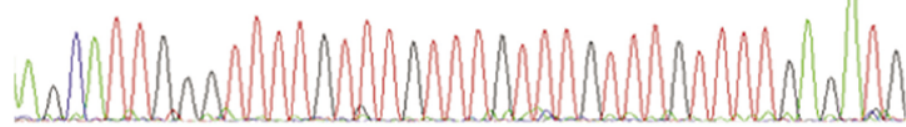

AGCATTGGGTTTTGTTTGTTTGTTTGTTTGTTTTGAGATG

C

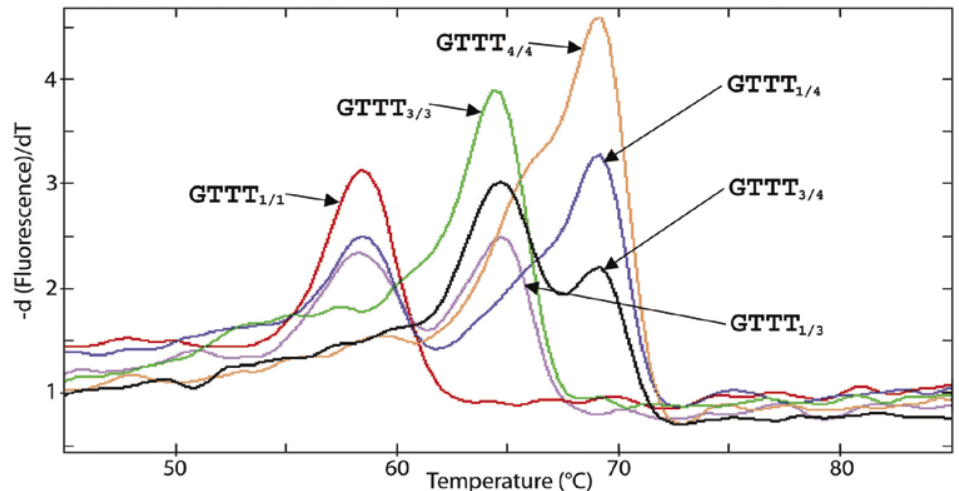

Figure 1. Design, DNA resquencing results and melting peaks of the assay of the IRGM tetranucleotide promoter oligopeat typing by fluorescence resonance energy tranfer (FRET). (A) The design for the detection of GTTT 4 (upper part) and GTTT 1 (lower part) is shown. While the sensor nucleotide (6-FAM labeled) matches GTTT 4 perfectly, a nonbinding oligonucleotide loop forms in sensor binding to GTTT $_{1}$, requiring a lower temperature $\left(T_{m}\right)$ for melting of the sensor from the matrix DNA. (B) DNA sequences (ABI Prism 6100; Applied Biosystems) of all genotypes occurring in the study population. (C) Melting peaks of all genotypes obtained by LightTyper/LightCycler-based genotyping. Melting data was plotted as a first negative derivation of fluorescence properties depending on $\mathrm{T}_{\mathrm{m}}(-\mathrm{dF} / \mathrm{dT})$. orientation and required for reaching the highest $\mathrm{T}_{\mathrm{m}}$. The sensor then would not be completely complementary to a matrix sequence containing the alternative $\mathrm{GTTT}_{1}$ or $\mathrm{GTTT}_{3}$ alleles and form nonbinding loops of GTTTGTTTGTTT nucleotides $\left(\mathrm{GTTT}_{1}\right.$; Figure $\left.1 \mathrm{~A}\right)$ or GTTT nucleotides $\left(\mathrm{GTTT}_{3}\right.$; not shown in Figure 1A), respectively. Forward (F) and reverse (R) PCR primers $\left(\mathrm{GTTT}_{4}\right.$ : $507 \mathrm{bp}$ ) and S/A oligonudeotides were 5'-CATCCTAACTCACCTGC TCTTCTA-3', 5'-TGTTAGAGATCAC CTCTGGCAA-3', 5' -TAAGCATTGG G T T T T G T T T G T T T G T T T G T T T G T T T T GA GA - 6 - FA M - 3' (6-FAM, 6-carboxyfluorescein) and 5'-Cy5-GGAGTCTTGCTCTGTTG CCAGGCTGGAG-phosphate-3' (Cy5) (6-FAM-and Cy5-labeled nucleotides were synthesized by biomers.net $\mathrm{GmbH}$, Ulm, Germany). Microtiter plates were prepared in the 384-well format with the Tecan GENESIS Workstation 200 pipetting instrument controlled by Gemini steering software v3.50 (Tecan, Crailsheim, Germany).

PCR conditions were optimized for the annealing temperature and $\mathrm{Mg}^{2+}$ concentration. The final PCR conditions (Primus 96 Thermocycler with 384-well block; MWG Biotech AG, Ebersberg, Germany) were $94^{\circ} \mathrm{C}$ for $3 \mathrm{~min}, 45$ cycles of $94^{\circ} \mathrm{C}$ for $20 \mathrm{~s}$, $60^{\circ} \mathrm{C}$ for $30 \mathrm{~s}, 72^{\circ} \mathrm{C}$ for $30 \mathrm{~s}$, with an extension at $72^{\circ} \mathrm{C}$ for $5 \mathrm{~min}$, with final concentrations of $3 \mathrm{mM} \mathrm{MgCl}_{2}, 0.1 \mu \mathrm{M}$ forward primer, $0.5 \mu \mathrm{M}$ reverse primer, $0.2 \mu \mathrm{M}$ sensor, $0.2 \mu \mathrm{M}$ anchor, $200 \mu \mathrm{M}$ dNTPs, 1 U Taq DNA polymerase, and 10 ng matrix DNA per reaction. The $10-\mu \mathrm{L}$ reaction mixtures were covered with $10 \mu \mathrm{L}$ of light mineral oil (Sigma Aldrich, Munich, Germany). The PCR success rate was $96.6 \%$. Prior to subsequent melting, amplicons were denatured for $3 \mathrm{~min}$ at $95^{\circ} \mathrm{C}$.

In the LightTyper instrument, samples were heated from $40^{\circ} \mathrm{C}$ to $80^{\circ} \mathrm{C}$ at a ramp rate of $0.1^{\circ} \mathrm{C} / \mathrm{s}$ with simultaneous continuous fluorescence monitoring. Melting data was plotted as a first negative derivation of fluorescence properties depending on $\mathrm{T}_{\mathrm{m}}(-\mathrm{dF} / \mathrm{dT})$. Different $\mathrm{T}_{\mathrm{m}}$ of perfect or reduced binding of the sensor oligonucleotide to the matrix DNA (Figure 1A) of different alleles allows for unambigu- 


\section{BioTechniques}

Connecting. Informing. Advancing. For 25 Years.

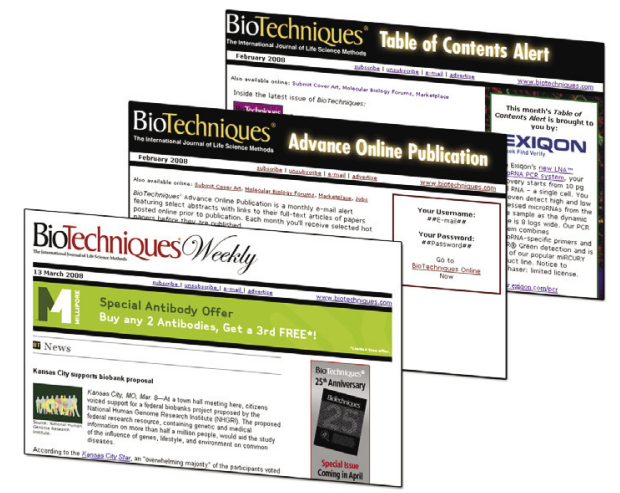

\section{BioTechniques E-Alerts}

\section{BioTechniques Weekly}

Weekly newsletter with industry insight for life scientists: news, new products, grants deadlines, employment opportunities, and events.

\section{Advance Online Publication (AOP)}

Monthly alert with select research papers published online ahead of the print.

\section{Table of Contents (TOC)}

Monthly alert listing current papers and their links allowing you to browse the latest issue immediately.

Register today at www.biotechniques.com/newsletters ously distinguishing homozygous from heterozygous individuals and for assessing the number of repeats. The melting curves of the IRGM promoter genotypes are shown in Figure 1C. Six genotypes were identified $\left(\mathrm{GTTT}_{1 / 1}\right.$, $n=364 ; \mathrm{GTTT}_{3 / 3}, n=85 ; \mathrm{GTTT}_{4 / 4}$, $n=303 ; \mathrm{GTTT}_{1 / 3}, n=425 ; \mathrm{GTTT}_{1 / 4}$, $n=717 ; \mathrm{GTTT}_{3 / 4}, n=292$ ). Alleles with two repeats were not observed at all in our study population, neither in the resequencing experiments nor in the analysis of melting curves.

Compared with the alternative techniques that are currently applied for oligotandem repeat typing, the LightTyper/LightCycler FRET-based system provides the advantages of high throughput, a reduced potential of DNA contamination, and less timeconsuming implementation and performance at a reasonable cost.

\section{ACKNOWLEDGMENTS}

The participation of the Ghanaian patients and control individuals is gratefully acknowledged, as well as the contributions of field workers, nurses and physicians involved in the recruitment of participants; the staff of the Kumasi Centre for Collaborative Research in Tropical Medicine (KCCR); and the excellent assistance of Emmanuel Abbeyquaye, Lincoln Gankpala, Birgit Muntau, Christa Ehmen and Gerd Ruge. This work was supported by the German Federal Ministry of Education and Research $(B M B F)$, German National Genome Research Network (NGFN1; Project 01GS0162; NGFN2, NIE-S17T20) and the BMBF Tuberculosis Research Network (Project 01KI0784).

\section{COMPETING INTERESTS STATEMENT}

The authors declare no competing interests.

\section{REFERENCES}

1. Bennett, C.D., M.N. Campbell, C.J. Cook, D.J. Eyre, L.M. Nay, D.R. Nielsen, R.P. Rasmussen, and P.S. Bernard. 2003. The LightTyper: high-throughput genotyping using fluorescent melting curve analysis. Biotechniques 34:1288-1295.
2. Lin, Z., J.G. Suzow, J.M. Fontaine, and E.W. Naylor. 2004. A high throughput betaglobin genotyping method by multiplexed melting temperature analysis. Mol. Genet. Metab. 81:237-243.

3. Simonsson, T. and R. Sjöback. 1999. DNA tetraplex formation studied with fluorescence resonance energy transfer. J. Biol. Chem. 274:17379-17383.

4. Simonsson, T., P. Pecinka, and M. Kubista. 1998. DNA tetraplex formation in the control region of c-myc. Nucleic Acids Res. 26:11671172.

5. Wang, Y., J. Ju, B.A. Carpenter, J.M. Atherton, G.F. Sensabaugh, and R.A Mathies. 1995. Rapid sizing of short tandem repeat alleles using capillary array electrophoresis and energy-transfer fluorescent primers. Anal. Chem. 67:1197-1203.

6. Malorny, B., E. Junker, and R. Helmuth. 2008. Multi-locus variable-number tandem repeat analysis for outbreak studies of Salmonella enterica serotype Enteritidis. BMC Microbiol. 8:84.

7. Butler, J.M. 2004. Short tandem repeat analysis for human identity testing. Curr. Protoc. Hum. Genet. 2004, Chapter 14:Unit 14.8.

8. Schmalzing, D., L. Koutny, D. Chisholm, A. Adourian, P. Matsudaira, and D. Ehrlich. 1999. Two-color multiplexed analysis of eight short tandem repeat loci with an electrophoretic microdevice. Anal. Biochem. 270:148-152.

9. Butler, J.M. 2006. Genetics and genomics of core short tandem repeat loci used in human identity testing. J. Forensic Sci. 51:253-265.

10. Oberacher, H., F. Pitterl, G. Huber, H. Niederstätter, M. Steinlechner, and W. Parson. 2008. Increased forensic efficiency of DNA fingerprints through simultaneous resolution of length and nucleotide variability by high-performance mass spectrometry. Hum. Mutat. 29:427-432.

11. Li, L., C.T. Li, R.Y. Li, Y. Liu, Y. Lin, T.Z. Que, M.Q. Sun, and Y. Li. 2006. SNP genotyping by multiplex amplification and microarrays assay for forensic application. Forensic Sci. Int. 162:74-79.

12. Reed, G.H., J.O. Kent, and C.T. Wittwer. 2007. High-resolution DNA melting analysis for simple and efficient molecular diagnostics. Pharmacogenomics 8:597-608.

Received 8 September 2008; accepted 6 November 2008.

Address correspondence to Christian G. Meyer, M.D., Ph.D., Bernhard Nocht Institute for Tropical Medicine, Bernhard Nocht Str. 74, 20359 Hamburg, Germany. email: c.g.meyer@bni.uni-hamburg.de

To purchase reprints of this article, contact: Reprints@BioTechniques.com 